\title{
BCCiN4
}

\section{NOTCHED STRENGTH OF SYMMETRIC ANGLE-PLY LAMINATES}

\author{
Lucas L. Vignoli $^{(1,2)}$, Jaime T.P. de Castro ${ }^{(3)}$
}

(1) Center for Nonlinear Mechanics, COPPE, Department of Mechanical Engineering, Universidade Federal do Rio de Janeiro, Brazil

(2) Department of Mechanical Engineering, Universidade Federal do Rio de Janeiro - Campus Macaé, Brazil

(3) Department of Mechanical Engineering, Pontifícia Universidade Católica do Rio de Janeiro, Brazil

https://doi.org/10.21452/bccm4.2018.03.03

\begin{abstract}
Even though composite materials are ever more used in structural applications, mainly due to their excellent strength/weight ratio, this material class is also intrinsically anisotropic, a condition that must be properly considered in theirs macroscopic analyses. It is well known that angle-plies instead of unidirectional laminates are preferable for practical applications, but it is demonstrated here that in addition damage initiation around notch borders in angleplies requires smaller nominal stresses than in unidirectional laminates, due to the uncoupling of normal/shear effects. To do so, the Stroh formalism and the Classical Laminate Theory are applied toghether with Tsai-Wu, Puck, and LaRC05 resistance criteria to analytically evaluate stress concentration effects introduced by circular holes in large anisotropic plates, and to estimate damage initiation around the hole border.
\end{abstract}

\section{INTRODUCTION}

Composite materials are widely used in various industrial areas for demanding structural applications, mainly because they have low specific weight and high strength. However, this material class is intrinsically anisotropic, especially unidirectional laminates that are the focus of this study. Since most real structures must contain notches for functional and/or assembly purposes, which induce stress/strain concentration effects that are the major cause for damage initiation, they must be properly analysed by suitable design procedures. This paper aims to discuss the analytical evaluation of such effects.

\section{FUNDAMENTAL ELASTICITY SOLUTIONS}

Considering a linear and elastic angle-ply laminate, the constitutive relation for its $P$-th ply in global coordinates is defined by [1] 


$$
\left[s_{i j}^{(g)}\right]_{P}=\left[s_{i j k l}^{(g)}\right]_{P}\left[e_{k l}^{(g)}\right]_{P}
$$

where $s_{i j}^{(g)}, s_{i j k l}^{(g)}$ and $e_{k l}^{(g)}$ are the stress, stiffness and strain tensors defined in global coordinates.

Using the Kirchhoff-Love kinematical assumption, the strain tensor can be defined as

$$
e_{i j}^{(g)}=\bar{e}_{i j}^{(g)}+x_{3}^{(g)} k_{i j}^{(g)}
$$

where $\bar{e}_{i j}^{(g)}$ is the midplane strain tensor and $k_{i j}^{(g)}$ is the curvature.

According to the Classical Laminate Theory (CLT), the forces and moments per unit of lengths are computed by the following equations

$$
\begin{aligned}
& N_{i j}^{(g)}=A_{i j k l}^{(g)} \bar{e}_{k l}^{(g)}+B_{i j k l}^{(g)} k_{k l}^{(g)} \\
& M_{i j}^{(g)}=B_{i j k l}^{(g)} \bar{e}_{k l}^{(g)}+D_{i j k l}^{(g)} k_{k l}^{(g)}
\end{aligned}
$$

where $N_{i j}^{(g)}=\int\left[s_{i j}^{(g)}\right]_{P} d x_{3}^{(g)}, M_{i j}^{(g)}=\int\left[s_{i j}^{(g)}\right]_{P} x_{3}^{(g)} d x_{3}^{(g)}, A_{i j k l}^{(g)}=\grave{o} s_{i j k l}^{(g)} d x_{3}^{(g)}, B_{i j k l}^{(g)}=\grave{o} s_{i j k l}^{(g)} x_{3}^{(g)} d x_{3}^{(g)}$ and $D_{i j k l}^{(g)}=\mathrm{ò}_{i j k l}^{(g)}\left(x_{3}^{(g)}\right)^{2} d x_{3}^{(g)}$. Restricting this study to symmetric laminates subjected to in-plane forces, $B_{i j k l}^{(g)}=0$ and $M_{i j}^{(g)}=0$. Hence, the laminate constitutive equation is

$$
N_{i j}^{(g)}=A_{i j k l}^{(g)} \bar{e}_{k l}^{(g)}
$$

Considering the average stress through the plate thickness, the average stiffness tensor is computed as

$$
\tilde{s}_{i j k l}^{(g)}=A_{i j k l}^{(g)} / t
$$

where $t$ is the plate thickness.

Once the equivalent elastic properties of the laminate are obtained by the CLT, to analyze stress concentration effects induced by a circular hole, it is necessary to evaluate the stress distribution along the hole border. Using the Stroh formalism, a very useul tool for the theory elasticity of anisotropic materials, the hoop stress along the hole border is written as [2]

$$
s_{11}^{(l)}=\mathbf{i}_{1}\left(\mathbf{G}_{1}^{(l)} \mathbf{t}_{2}+\mathbf{G}_{3}^{(l)} \mathbf{t}_{1}\right)-\mathbf{i}_{2}\left(\mathbf{G}_{1}^{(l)} \mathbf{t}_{1}-\mathbf{G}_{3}^{(l)} \mathbf{t}_{2}\right)
$$

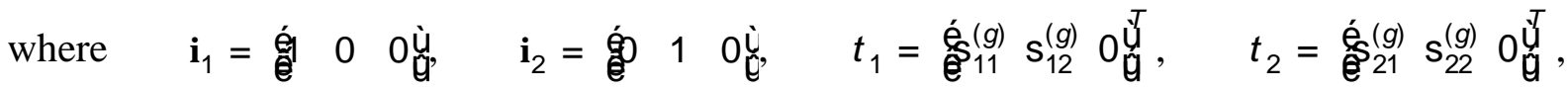

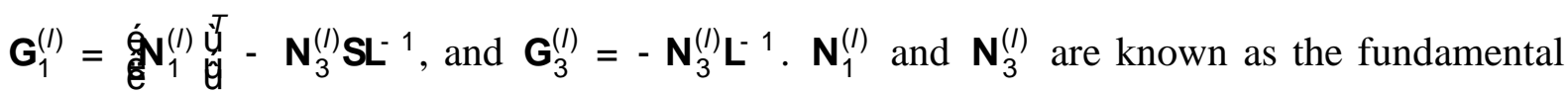
elasticity matrices, and $\mathbf{S}$ and $\mathbf{L}$ are the Barnet-Lothe tensors. These quantities are defined as

$$
\begin{aligned}
& \mathbf{N}_{1}^{(l)}=-\left[\mathbf{T}^{(l)}\right]^{1}\left[\mathbf{R}^{(l)}\right]^{\top} \\
& \mathbf{N}_{3}^{(l)}=-\mathbf{R}^{(l)}\left[\mathbf{T}^{(l)}\right]^{1}\left[\mathbf{R}^{(l)}\right]^{\top}-\mathbf{Q}^{(l)}
\end{aligned}
$$




$$
\begin{aligned}
& \mathbf{S}=\frac{1}{p} \grave{\mathrm{O}}_{0}^{p} \mathbf{N}_{1}^{(l)} d q \\
& L=\frac{1}{p} \grave{\mathrm{O}}_{0}^{p} \mathbf{N}_{3}^{(l)} d q
\end{aligned}
$$

where $Q_{i k}=s_{i 1 k 1}^{(g)}, R_{i k}=s_{i 1 k 2}^{(g)}, T_{i k}=s_{i 2 k 2}^{(g)}$ and

$$
\begin{aligned}
& \mathbf{Q}^{(l)}=\mathbf{Q} \cos ^{2} q+\left(\mathbf{R}+\mathbf{R}^{T}\right) \sin q \cos q+\mathbf{T} \sin ^{2} q \\
& \mathbf{R}^{(l)}=\mathbf{R} \cos ^{2} q+(\mathbf{T}-\mathbf{Q}) \sin q \cos q+\mathbf{R}^{T} \sin ^{2} q \\
& \mathbf{T}^{(l)}=\mathbf{T} \cos ^{2} q-\left(\mathbf{R}+\mathbf{R}^{T}\right) \sin q \cos q+\mathbf{Q} \sin ^{2} q
\end{aligned}
$$

\section{FAILURE CRITERIA}

According to Soden et al. [3], the models that better matched with experimental results from the worldwide failure exercise known as WWFE-1 (a round-robin to experimentally evaluate the modeling of composite failures) were Tsai-Wu, Puck, and Cuntze. Among these, Cuntze's model does not need to be discussed here because for damage initiation, this paper aim, it is quite similar to Puck's model. Kaddour and Hinton [4] pointed out that Carrere's and the LaRC05 models generated the better predictions for the data obtained in the following WWFE-II. However, just the LaRC05 is studied here, because Carrere's model includes micromechanics approaches, which are not within this paper scope. Thus, Tsai-Wu, Puck, and LaRC05 failure criteria are presented next, to be later used to estimate the strength of unnotched and notched laminated plates. Note that despite Puck and LaRC05 are able to indicate which constituint fails, they do not consider any explicit micromechanical approach.

\subsection{Tsai-Wu Criterion}

Tsai and $\mathrm{Wu}$ [5] proposed a well known and widely used generic failure criterion for anisotropic materials based on a polynomial function which is simple to implement and compute, albeit it does not distinguish failure mechanisms. For plane stress conditions, their damage function is described by

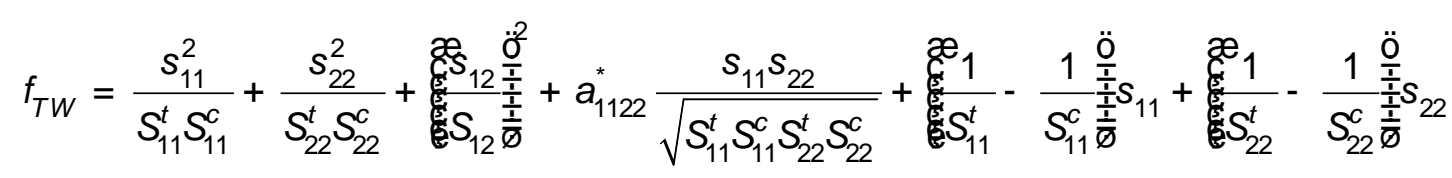

where $a_{1122}^{*}=-1$ is recommended in absence of experimental data for proper calibration. For Eq.(15) and for all the other models, failure is assumed to take place when their damage functions equal 1.

\subsection{Puck Criterion}

The Puck criterion [6] use different approaches to model different failure mechanisms in composite materials, recognizing the difference between fiber and matrix failures, as well as different tension and compression effects. For fibers under tensile loads, using the maximum normal stress theory as a basis, his damage function is given by 


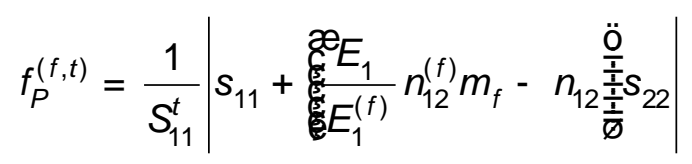

where $m_{f}=1.3$ is recommended for glass fibers.

Considering the shear influence on the fibers instability when they are compressed, the function to describe fiber damage under compression is

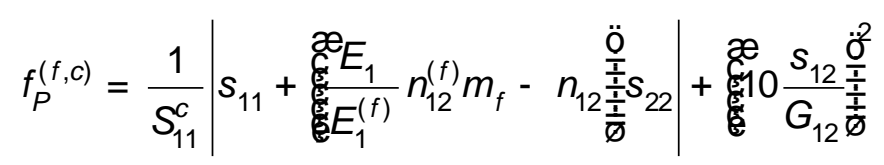

For modeling matrix failures, the key-point of Puck's model is to identify and to evaluate damage on a critical plane, similar to the Coulomb-Mohr criterion. For plane stress, three different failure modes are modelled: mode A, when the matrix is in tension and the critical plane is parallel to the fibers; mode $\mathrm{B}$, that has the same critical plane than mode $\mathrm{A}$ but the matrix is compressed; and mode $\mathrm{C}$, where the matrix is also in compression, however the critical plane inclination is $g_{C}=\operatorname{acos}\left\{\left[\left(1 / 2\left(1+p_{12}^{c}\right)\right)\left(\left(S_{23}^{(23)} S_{12} / S_{12} s_{22}\right)^{2}+1\right)\right]^{1 / 2}\right\}$, where for glass fibers $p_{12}^{c}=0.25$ and $S_{23}^{(23)}=S_{12} / 2 p_{12}^{c}\left[\left(1+2 p_{12}^{c} S_{22}^{c} / S_{12}\right)^{1 / 2}-1\right]$. The damage functions for theses modes are

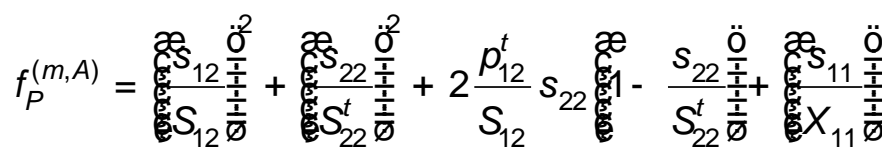

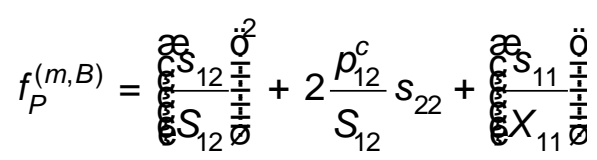

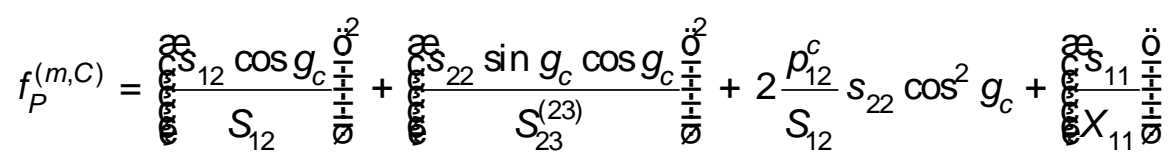

where $p_{12}^{t}=0.30$ is recommended for glass fiber, $6<n<8$ ( $n=8$ is adopted here) and $X_{11}=1.1 S_{11}^{t}$ if $S_{11}{ }^{3} 0$ or $X_{11}=-1.1 S_{11}^{c}$ otherwise.

\subsection{LaRC05 Criterion}

This failure criterion has been developed since 2000's and has been proven to be able to generate quite accurate predictions. Despite Pinho et al. [7] data indicates a nonlinear behavior of the transverse elastic and of the shear moduli, both are assumed linear here, since just initial damage is analyzed and these properties becomes nonlinear for higher strain levels.

For the matrix failure, the Puck model is used as a basis, mainly because of its use of a critical plane concept. Therefore, just one damage equation is used, but it requires a critical plane search. The matrix damage function is

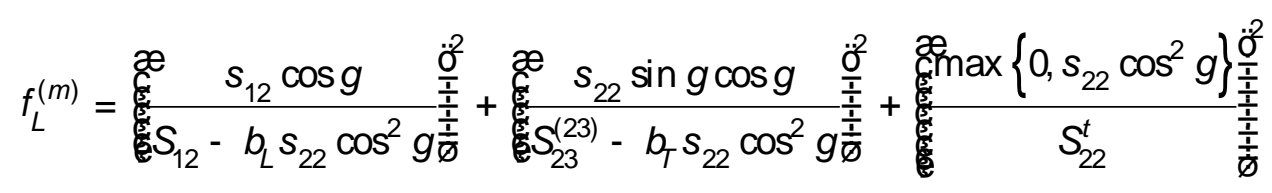


where $b_{L}$ and $b_{T}$ are parameters that can be experimentally calibrated, $g$ is the critical plane angle, and $S_{23}^{(23)}$ is the shear strength on this plane. According to Dávila et al. [8], $b_{L}=0.082$ and $q_{0}=53^{\circ}$, used in $b_{T}=-1 / \tan 2 q_{0}$ and $S_{23}^{(23)}=S_{22}^{c} \cos q_{0}\left(\sin q_{0}+\cos q_{0} / \tan 2 q_{0}\right)$, are good approximations if no experimental data is available.

For modeling the failure of fibers under tensile loads, the maximum normal stress theory is assumed valid, resulting on the following damage function

$$
f_{L}^{(f, t)}=s_{11} / S_{11}^{t}
$$

The main advantage of this model when compared to the Puck's one is the modelling of fibers failure when these are compressed, because it considers in a more sophisticated way the fibers instability. If all the fibers are initially aligned, a rotation $f$ along the plane $x_{2}-x_{3}$ is necessary to search the critical plane. Once this procedures is finished, the stress components in this plane are computed as $s_{i j}^{(f)}=l_{i k} l_{j l} s_{k l}$. Nevertheless, an initial misalignment of the fibers is an intrinsic issue for real manufacturing processes, and must be considered in failure analyses, because it may have a major contribution for instability failures due to the fibers initial deflection. Hence, in the presence of an initial misalignment angle $j_{0}$, the total misalignment is computed considering the initial value induces the shear contribution:

$$
j=\operatorname{sign}\left(s_{12}^{(f)}\right) j_{0}+2 e_{12}^{(m i s)}
$$

where

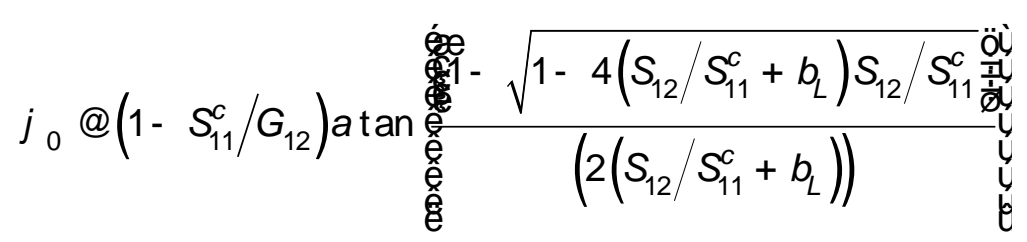

Once the misalignment direction is obtained, the stress components in this planes are computed with the transformation $s_{i j}^{(m i s)}=l_{i k} l_{j l} s_{k l}^{(f)}$. At last, the damage function of the fibers in compression is defined by

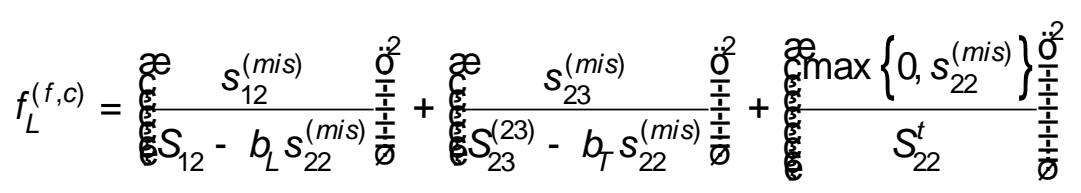

\section{RESULTS AND DISCUSSION}

Considering the unidirectional $[\alpha]_{n}$ and angle-ply $[ \pm \alpha]_{n s}$ laminates, their tensile $\left(S_{F P F}^{t}(\alpha)\right)$ and compressive $\left(S_{F P F}^{c}(\alpha)\right)$ strength variations according to the fiber-to-load angles predicted by Tsai-Wu, Puck, and LaRC05 criteria are plotted in Fig. 1 for an unnotched plate. Notice the very high effect of the fiber angle with respect to the load direction on the laminate strength predicted by all criteria, a major issue when dealing with laminates. This is the main 
reason for their peculiar stress concentration behavior, studied next. Figure 1 illustrates how matrix failure usually is the dominant mechanism. For the single layered laminate, the tensile strength decrease more than $80 \%$ when the fibers angle $\alpha$ changes from $0^{\circ}$ to $15^{\circ}$, a major effect that highlights well how directional such composite materials are.
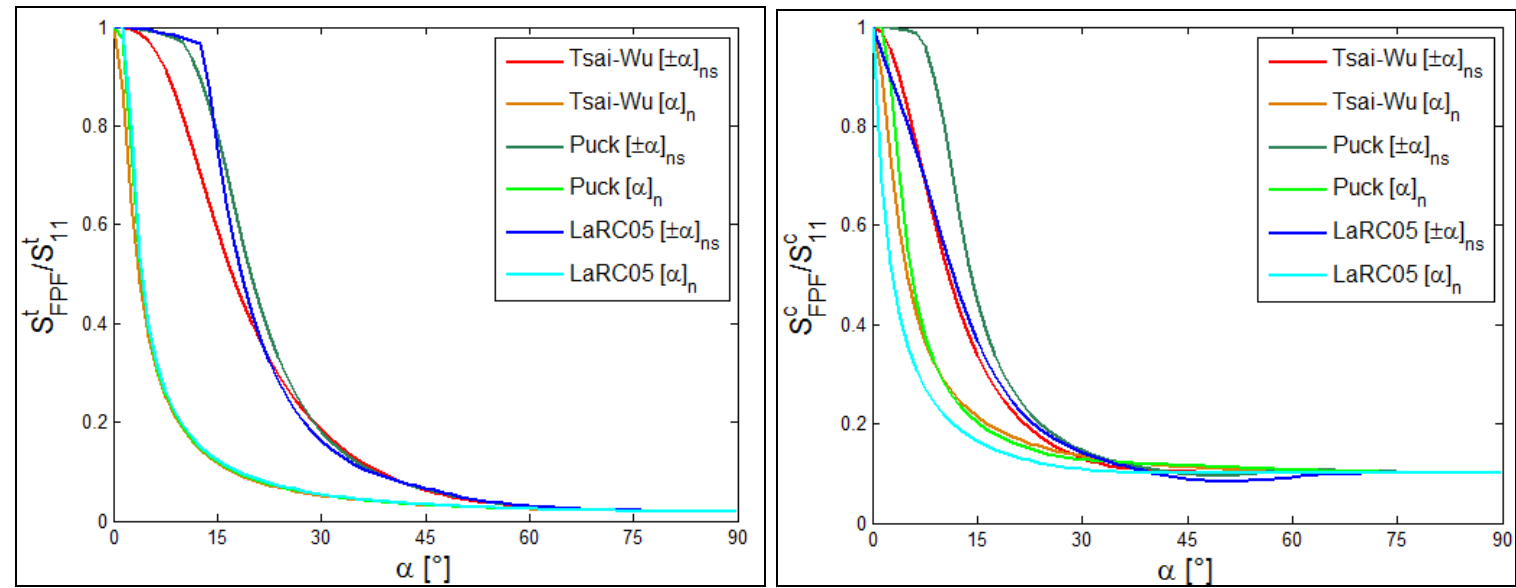

Figure 1. Variation of the unnotched laminate plate FPF strength prediction as function of the fiber orientation angle $\alpha$ with respect to the uniaxial load direction, $S_{F P F}^{t}(\alpha) / S_{11}^{t}$ and $S_{F P F}^{c}(\alpha) / S_{11}^{c}$, where $S_{11}^{t}$ and $S_{11}^{c}$ are the reference tensile and compressive strengths measured when the fibers are aligned with the load, i.e. when $\alpha=0$.

This simple analysis, still without considering any stress concentration effects, helps to understand the results presented next. In particular, those that show that the critical point along a circular hole border in anisotropic plates in general is not the point where the stress concentration peaks occur. Instead, it is the point that maximizes the ratio between the local stress and the smallest (anisotropic) material strength along the notch border. This usually is a major issue when designing notched components made of anisotropic materials, since their behaviour can be very different from the well-known behaviour of notched isotropic materials.

Additionally, the LaRC05 model indicated that the strength of angle-ply laminates may be smaller than that of unidirectional laminates with the same angle $\alpha$. This conclusion applies for fiber-to-load angle $\alpha$ between $45^{\circ}$ and $60^{\circ}$, and it is a consequence of the shear influence in the longitudinal compression resistance modelled by the LaRCO05 criterion.

First, using the CLT, it is possible to conclude that angle-ply laminates do not couple shear and normal stress and strains, because the elements responsible for this physical coupling on the equivalent stiffness and equivalent compliance tensors are null, unlike what happens for $[\alpha]_{n}$ if $\alpha \neq 0^{\circ}, \pm 90^{\circ}$, a result that is valid for any angle-ply laminate. Notice that $[+\alpha]_{n}$ and $[-\alpha]_{n}$ are two different laminates, and that $[ \pm \alpha]_{n s}$ is one laminate that contains laminas with both angles, $+\alpha$ and $-\alpha$.

First ply failure (FPF) notched strength predictions for single-layered and angle-ply laminates are presented in Fig. 2. Clearly, the $[0]_{n}$ and $[90]_{n}$ laminates must be equivalent to $[ \pm 0]_{n s}$ and $[ \pm 90]_{n s}$, respectively, but their strength variation according to the fiber-to-load angle $\alpha$ have a significantly different behavior. For tension or for compression, the strength curves have a smoother shape for single layered laminates, unlike what happens for un-notched plates. Figure 1 shows that in notch-free laminates, the angle-plies strength variations are smoother. These results are justified by the vanishing effect of the normal-shear coupling in equivalent homogeneous materials, where the stress concentration is maximized, e.g. for $[\alpha]_{\mathrm{n}}$ where the stress concentration is maximized if $\alpha=0^{\circ}, \pm 90^{\circ}$. 

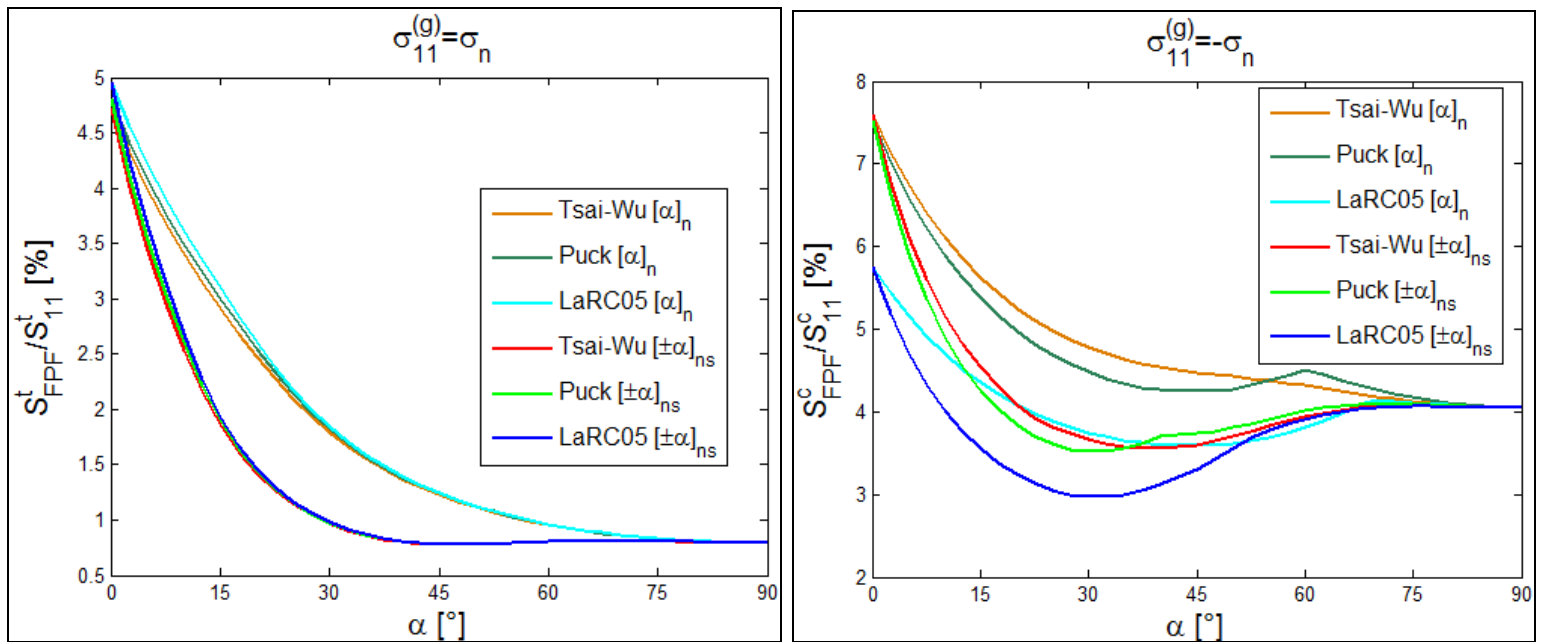

Figure 2. Prediction of $S_{F P F}^{t} / S_{11}^{t}$ and $S_{F P F}^{c} / S_{11}^{c}$ for the holed orthotropic plate FPF strength under tension and under compression loads by the 3 failure criteria, for angle-ply laminates, as a function of the fibers-to-load angle $\alpha$.

While the stress concentration around the border of a circular hole in isotropic plates with uniaxial load ranges between -1 and 3 for isotropic materials, for the single-layered laminate studied it ranges between -4 and 7 [9]. For angle-ply laminates the stress concentration effect is even more pronounced. By this fact, the ration between notched and unnotched strengths presented in Fig. 2 is smaller than 10\% for all cases.

\section{CONCLUSIONS}

This paper presented a brief review of some theoretical approaches for stress analyses of anisotropic materials, in particular laminated composites (Stroh Formalism and CLT). Three failure theories are studied here, using as basis the WWFE results and their recommendations: Tsai-Wu, Puck, and LaRC05. Then these techniques are used to predict the behaviour of simple notched plates. Large plates made by single layered unidirectional laminates with circular holes had a FPF strength decrease estimation of more than 95\% and 90\% for uniaxial tension and compression, respectively. These results, which differ significantly from the behaviour of isotropic plates, become even more pronounced depending of the fiber orientation. Notched angle-ply laminates have a smaller FPF strength for most of the cases analyzed, on the contrary of unnotched plates. For notched plates, the strength of symmetric angle-ply laminates is usually smaller than for unidirectional laminate due to the increase of stress concentration effects, what is the opposite of the observed for unnotched plates.

\section{ACKNOWLEDGEMENTS}

L.L. Vignoli would like to acknowledge the support of the Brazilian Research Agencies CNPq and CAPES, and ffrom PUC-Rio.

\section{REFERENCES}

[1] Jones, R.M., “Mechanics of Composite Materials”. 2nd Edition. Taylor \& Francis Editions, 1998.

[2] Hwu, C., “Anisotropic Elastic Plates”. Springer, 2009.

[3] Soden, P.D., Kaddour, A.S., Hinton, M.J., Recommendations for designers and researchers resulting from the world-wide failure exercise. Compos. Sci. Technol. 2004;64:589-604. 
[4] Kaddour, A.S., Hinton, M.J., Maturity of 3D failure criteria for fibre reinforced composites: Comparison between theories and experiments: Part B of WWFE-II. J. Compos. Mater. 2013;47:925-966.

[5] Tsai, S.W., Wu, E.M., A general theory of strength for anisotropic materials. J. Compos. Mater. 1971;5:58-80.

[6] Puck, A., Schürmann, H., Failure analysis of FRP laminates by means of physically based phenomenological models. Compos. Sci. Technol. 1998;58:1045-1067.

[7] Pinho, S.T., Darvizeh, R., Robinson, P., Schuecker, C., Camanho, P.P., Material and structural response of polymer-matrix fibre-reinforced composites. J. Compos. Mater. 2012;46:23132341.

[8] Pinho, S.T., Dávila, C.G., Camanho, P.P., Iannucci, L., Robinson, P., Failure Models and Criteria for FRP Under In-Plane or Three-Dimensional Stress States Including Shear NonLinearity. NASA/TM-2005-213530, 2005.

[9] Vignoli, L.L., Castro, J.T.P., Meggiolaro, M.A., Stress Concentration Issues in Unidirectional Laminates, submitted to Composite Structures. 